\title{
Optomizing Transfection Efficiency of Cervical Cancer Cells Transfected by Cationic Liposomes Lipofectamine ${ }^{\mathrm{TM}} 2000$
}

\author{
Fei Huang ${ }^{1 \&}$, Feng Zhao ${ }^{1 \&}$, Li-Ping Liang ${ }^{1}$, Mei Zhou ${ }^{1}$, Zhi-Ling Qu $^{2}$,Yan-Zhen \\ $\mathrm{Cao}^{1}$, Chen Lin ${ }^{3 *}$
}

\begin{abstract}
Background: Currently, cationic liposome has become the commonly used vehicles for gene transfection. Furthermore, one of the most significant steps in microRNAs expression studies is transferring microRNAs into cell cultures successfully. In this study we aim to approach the feasibility of transfection of cervical cancer cell lines mediated by liposome and to obtain the optimized transfection condition for cervical cancer cell lines. Materials and Methods: Lipofectamine ${ }^{\mathrm{TM}} 2000$ as the carrier, miR-101 mimic was transfected into Hela cells and Siha cells. Using green fluorescent protein as reporter gene, to set different groups according to cell seeding density, the amount of miRNA, miRNA and the proportion of Liposomes, Whether to add serum into medium to study their impact on the liposomal transfection efficiency. Finally, MTT assay was used to analyze the relative minimal cell toxicity of liposome reagents. $\underline{\text { Results: }}$ The seeding density of Hela cell line and Siha are $1.5 \times 10^{4}$ (per well of 24 well plates), miRNA amount is 1 ul of both, the ratio of miRNA and liposome is 1:0.5 of Hela cell line; 1:0.7 of Siha cell line respectively, after 24 hours we can get the highest transfection efficiency. Compared with serum medium, only Siha cells cultured with serum-free medium obtained higher transfection efficiency before transfection $(\mathrm{P}<0.01)$.MTT assay showed that according to the above conditions which has the lowest cytotoxicity. Conclusions: The method of Liposome to transfected is a suitable way and it can be an efficient reagent for miRNA delivery for Hela cells and Siha cells in vitro. It may serve as a reference for the further research or application.
\end{abstract}

Keywords: MicroRNA - cervical cancer cell - cell transfection liposome - transfection efficiency

Asian Pac J Cancer Prev, 16 (17), 7749-7754

\section{Introduction}

In most studies, gene expression in cells under in vitro conditions is shown to provide a powerful tool for studying key interactions in some cells. Cationic liposomes as a simple operation, reproducible gene transfer vector has a far-reaching potential of applications in the medical research (Xiong et al., 2011). However, different cell types require slightly different transfection conditions of cationic liposomes. There are a few considerations that should be highlighted for transfection procedures: Cell seeding density ,the amount of miRNA, Liposomes and the proportion of miRNA, the time of transfection (Liang et al., 2015). All of them are the key parameters to maximize transfection efficiency and relatively minimal of cell toxicity.

It is generally known that Hela cell line is adenocacinoma cell and Siha cell is squamous cell of human cervical. These two cell lines play an essential role in cancer research, biological or cell culture experiments (Asgharian et al., 2014; Lee et al., 2015; Pradines et al., 2015). However, how to make the efficiency transfection of Hela cells and Siha cells to be maximized is a general concerned problem for researchers.

MicroRNAs (miRNAs) are a group of endogenously expressed, non-coding small RNAs (20-25 nucleotides in length) known to negatively regulate gene expression by suppressing translation or decreasing the stability of miRNAs by directly binding to the 3'-untranslated region (3'-UTRs) of target gene (Geusens et al., 2009; Gaedcke et al., 2012; Takahashi et al., 2015). Previously, we found that miR-101 is significantly down-regulated in cervical cancer tissues from Uyghur women.

The objectives of this study are to explore the feasibility of liposome transfected cervical cancer cells, obtain the optimal transfection conditions of liposome transfected cervical cancer cells, use it to establish liposome transfection mediated of cervical cancer cell system. 


\section{Materials and Methods}

Cell culture: The HeLa cells and Siha cells were obtained from the Cell Bank of Shanghai. Cells were grown in DMEM and 1640 medium (Gibco) and with 10\% fetal bovine serum, $50 \mathrm{U} / \mathrm{mL}$ of penicillin, and $50 \mathrm{mg} / \mathrm{mL}$ of streptomycin. At $37^{\circ} \mathrm{C}$ in a humidified atmosphere of $95 \%$ air and $5 \% \mathrm{CO}_{2}$.

Cells transfected: The day before transfection, Stated growing Hela cells about $1.5 \times 10^{4}$ was inoculated to a 24-well plate, Continue to train, Reference instructions of Lipofectamine $\mathrm{TM}^{\mathrm{TM}} 2000$. The human miR-101 duplex mimic (miR-101) were designed and provided by Invitrogen .

Choice of cell seeding concentration: Logarithmic growth phase cells were seeded in 24-well plates, After counted, as the different cell inoculation concentrations to group in the 24 -well plate. Were $0.5 \times 10^{4}, 1.0 \times 10^{4}, 1.5 \times 10^{4}$, $2 \times 10^{4}$. The gradient groups of each hole were repeated 3-4 times, Other conditions of transfection are the same.

\section{Choose the amount of miRNA}

Cells were seeded per well in 24 well plates as the optimal cell density of inoculum obtained above, according to the amount of miRNA in each well. Each group were: $0.5 \mathrm{ul}, 0.75 \mathrm{ul}, 1 \mathrm{ul}, 1.25 \mathrm{ul}$, the amount of liposomes $1 \mu \mathrm{l}$. The gradient groups of each hole were repeated 3-4 times, Other conditions of transfection are the same.

\section{The ratio between miRNA and liposome}

The amount of miRNA was obtained in the abovedescribed optimum amount, The proportion with miRNA and liposomes were 1: $0.5,1: 0.7,1: 0.9$ and 1: 1.1 . The gradient groups of each hole were repeated 3-4 times, Other conditions of transfection are the same.

\section{The time of transfection}

Set a different medium change time $(6,1224$ and $48 \mathrm{~h})$ to optimize the best liposomes transfection time. The gradient groups of each hole were repeated 3-4 times, Other conditions of transfection are the same. Other conditions: cell seeding density, the proportion of the amount of miRNA, miRNA and liposome ratio selection, the best results are obtained in the above experiment.

\section{The selection of serum}

Divided two cases into before transfection with serogroup and no serogroup, Before transfection with serogroup ( Rinse cells before transfection, then swapped DMEM/F12 medium which containing a volume fraction of $10 \%$ fetal calf serum.) and no serogroup (Rinse cells before transfection, then swapped DMEM/1640 medium which without serum) .Other transfection conditions are the same.

\section{Detection of GFP expression and transfection efficiency evaluation}

Transfection after 24 hours, Use $200 \times$ an inverted fluorescence difference microscope observation cell morphology, The same field to detect the expression of GFP, To count 5 perspection cell number of green fluorescent and the total cell number, seeking GFP expression rate. Transfection rate $=$ cell number of green fluorescent / total number of cells.

\section{Cytotoxicity test}

The MTT method was used to estimate cell viability. We use the optimal transfection conditions of the above experiments to MTT assay.MTT (BIO-BOX) was added to each well followed by a 4-hour incubation at $37^{\circ} \mathrm{C}$. After removing the media, dimethyl sulfoxide was added to each well for solubilizing the formazan formed. After 30 minutes at room temperature, the plates were scanned spectrophotometrically with a microplate reader set at 490 nm for measuring the absorbance.

\section{Statistical analysis}

All data are expressed as mean \pm SD with at least three replicates for each experiment. Statistical analysis using SPSS 17.0 (SPSS Inc.). Data were compared between groups using single-factor analysis of variance, Pairwise differences between multiple groups were compared using SNK-q test. Results were considered statistically significant when the $P$ value was less than 0.05 . The expression rate in chart data is the average of triplicate experiments

\section{Results}

Different densities of inoculum, different effects of transfection

To get the best transfection conditions, first need to explore the influence of different cell densities in inoculum. The results shown in Figure 1and Figure2, After 24 hours transfection, observed, it is showed that in the same amount of miRNA and Lipofectamine ${ }^{\mathrm{TM}} 2000$, Cells were seeded concentration of $1.5 \times 10^{4}$ cells per well, comparing the highest transfection efficiency with other groups, $(\mathrm{P}<0.05$ or $\mathrm{P}<0.01)$.

The amount of miRNA not only enhance the efficiency of transfection, but also save the consumption of reagent

After 24 hours transfection, observed, the results show that when the amount of miRNA is $0.5 \mathrm{ul}$, Obviously there is not has a high transfection efficiency; while, When the amount of miRNA is $1 \mathrm{ul}$ or $1.25 \mathrm{ul}$, the results of transfection do not change substantially. This shows

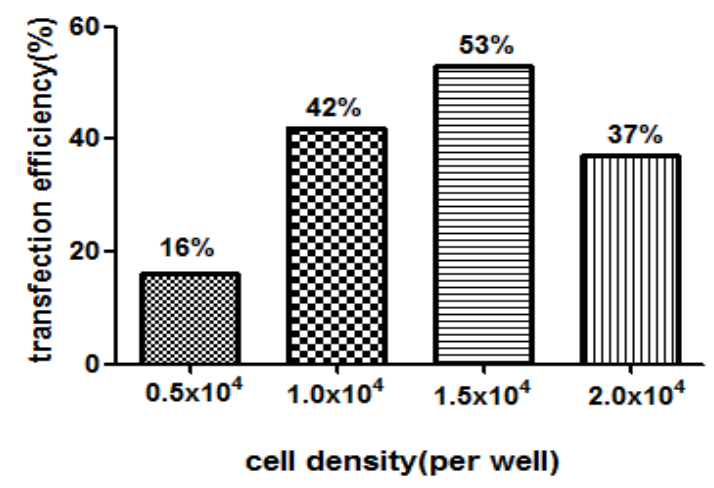

Figure 1. Effect of Hela Cell Density on Transfection Effciency 
that when cells were seeded at the same density, after achieve the optimum amount of miRNA, The efficiency of transfection no longer following the amount of miRNA to increases, excessive amount of miRNA will lead to a waste of reagents. Thus, it is most suitable when the amount of miRNA is 1ul (Figure 3 and Figure 4).

The ratio between miRNA and liposome impact on transfection efficiency

The result showed that the ratio between miRNA and liposome is 1: 0.5 of Hela cell line; 1:0.7 of Siha cell line respectively, which are the best ratio when transfected, Ratio is too small will cause the amount of Lipofectamine ${ }^{\mathrm{TM}} 2000$ not enough to complete the

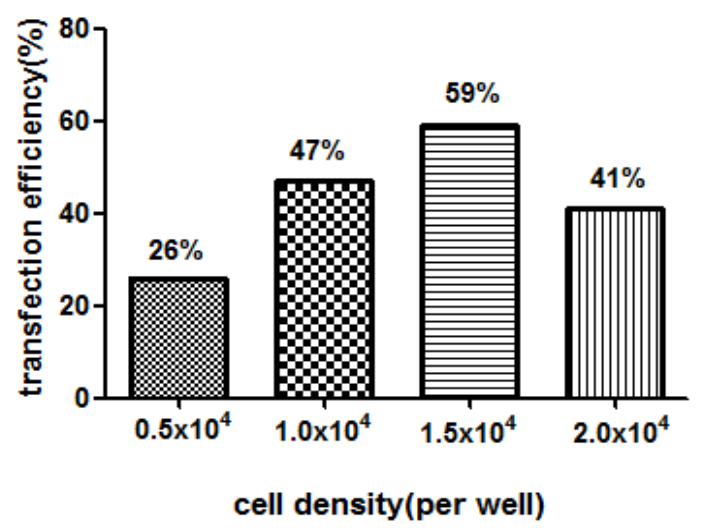

Figure 2. Effect of Siha Cell Density on Transfection Effciency

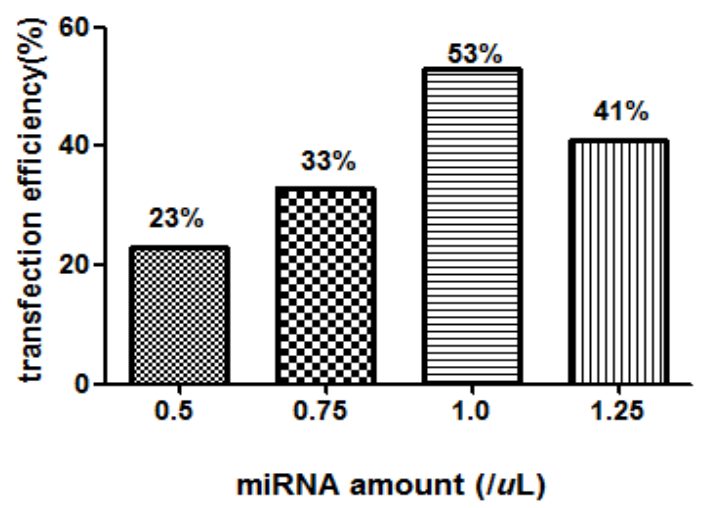

Figure 3. Effect of miRNA Amount on Transfection Effciency (Hela)

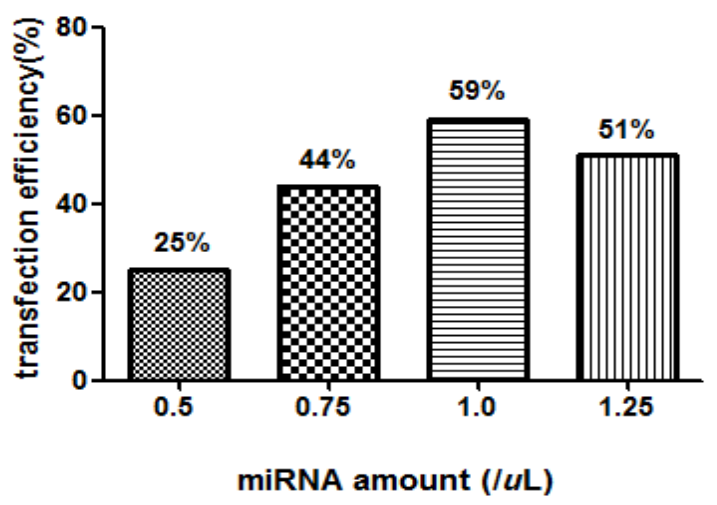

Figure 4. Effect of miRNA Amount on Transfection Effciency (Siha) transfection; When the ratio is maximum, the death rate of cell is also increased (Figure 5 and Figure 6).

The time of transfection impact on transfection efficiency

When all the conditions of optimization are the same , Transfection time was 24 hours is the best (Figure 7 and Figure 8).

\section{Serum can impact on transfection efficiency}

No serum before transfection group than those with serum group has a high transfection efficiency only exist in Siha cell. There were significant differences $(\mathrm{P}<0.01)$; After 6 hours for transfection, the efficiency of transfection is not statistically significant difference between no serum

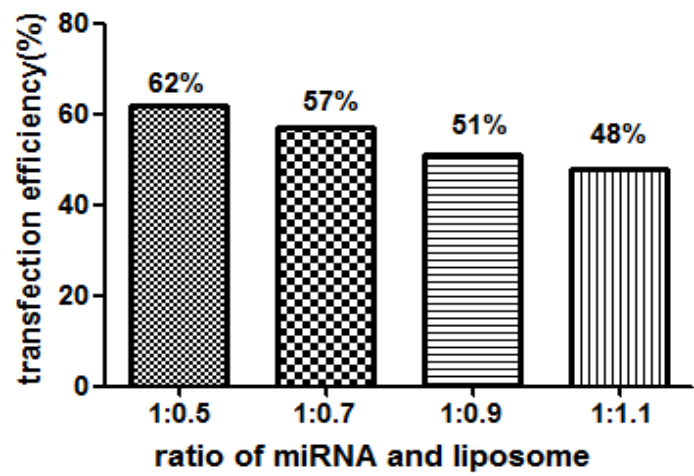

Figure 5. Effect of ratio of miRNA and liposnme on Transfection Effciency (Hela)

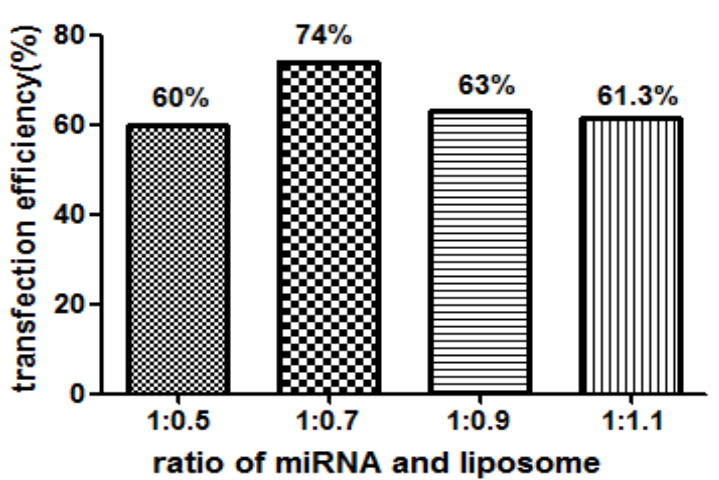

Figure 6. Effect of ratio of miRNA and liposnme on Transfection Effciency (Siha)

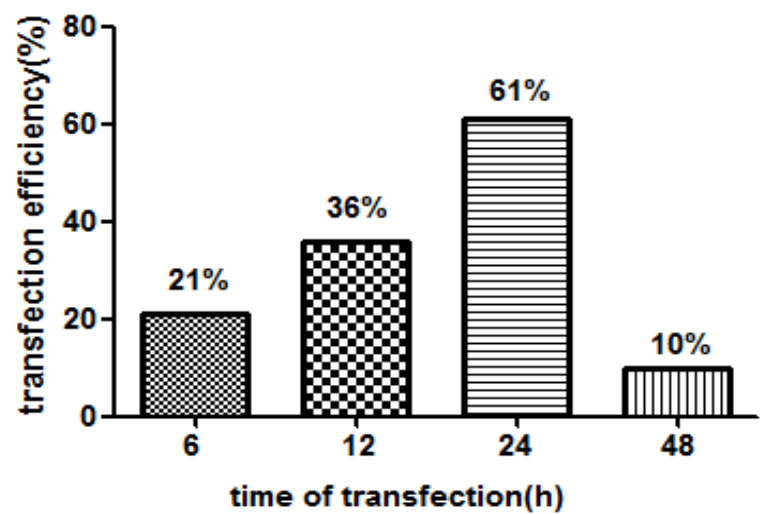

Figure 7. Effect of time on Transfection Effciency (Hela) 


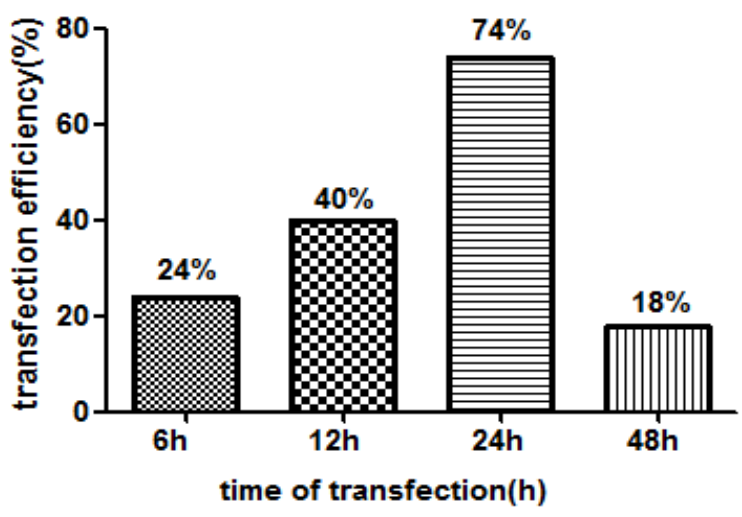

Figure 8. Effect of time on Transfection Effciency (siHa)

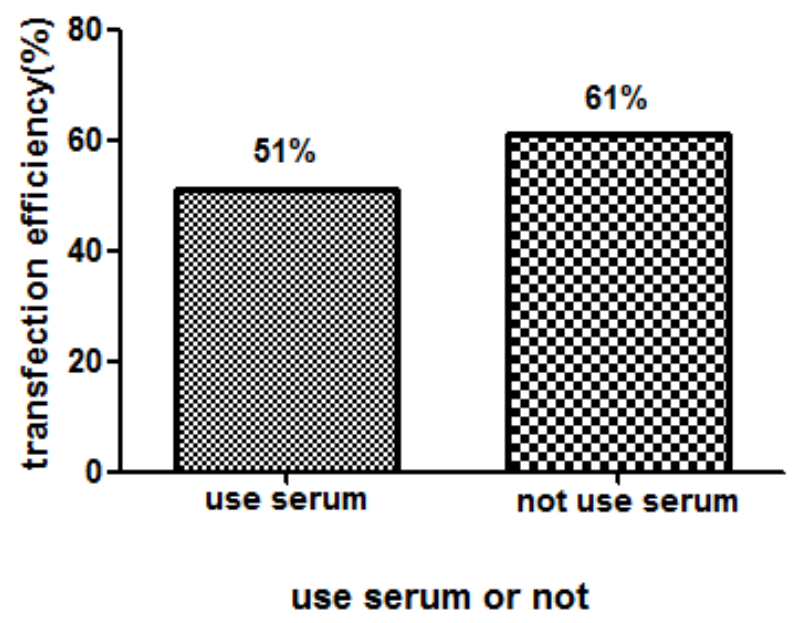

Figure 9. Effect of Serum on Transfection Effciency (Hela)

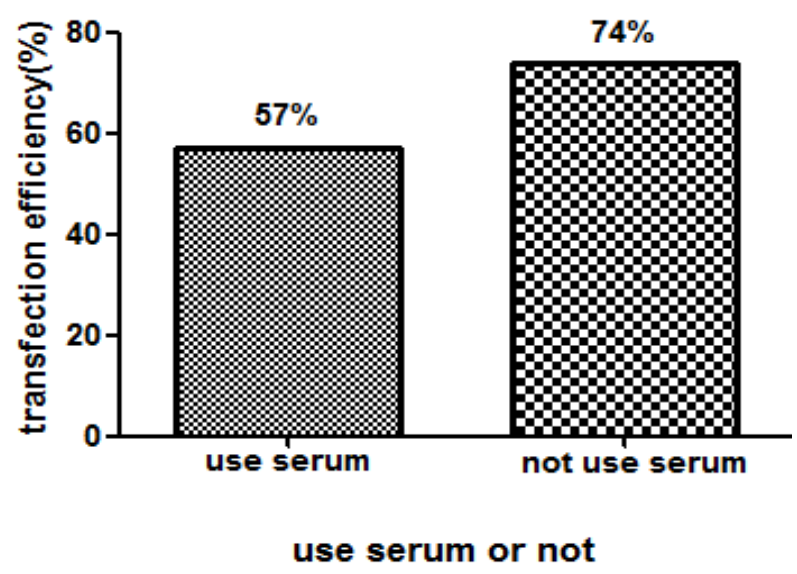

Figure 10. Effect of Serum on Transfection Effciency (Siha)

group and serum group $(\mathrm{P}>0.05)$. But for Hela, there was no statistically significant difference between two groups of results. (Figure 9 and Figure10)

\section{Cytotoxicity test}

The growth ability of Hela and Siha cell was determined by MTT assay in cells transfected with miR-101 mimic for 24,48 , and $72 \mathrm{~h}$. There was no signifcant difference in the proliferation rate at the beginning of transfection. At the time point of $0,24,48$, and $72 \mathrm{~h}$ posttransfection of miR-101 mimics, the proliferation were decreased

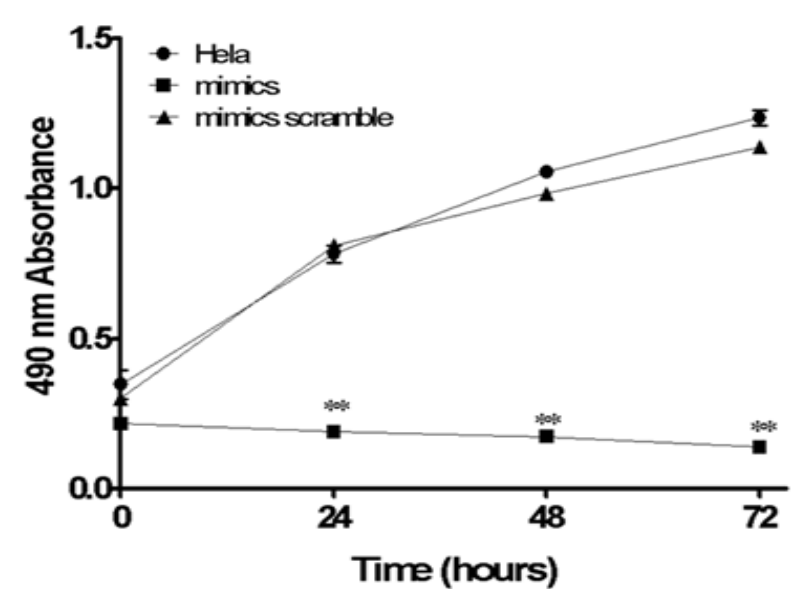

Figure 11. The Growth Ability of Hela Cell was Determined by MTT Assay

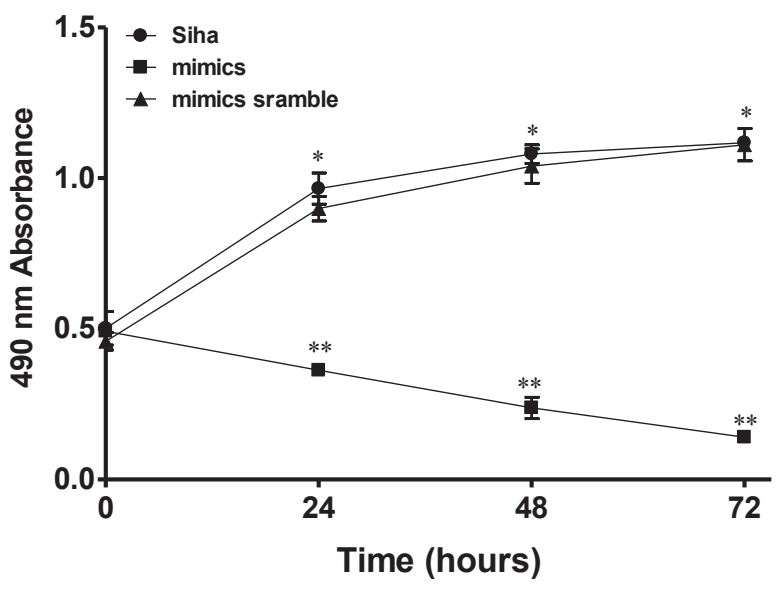

Figure 12. The Growth Ability of Siha Cell was Determined by MTT Assay

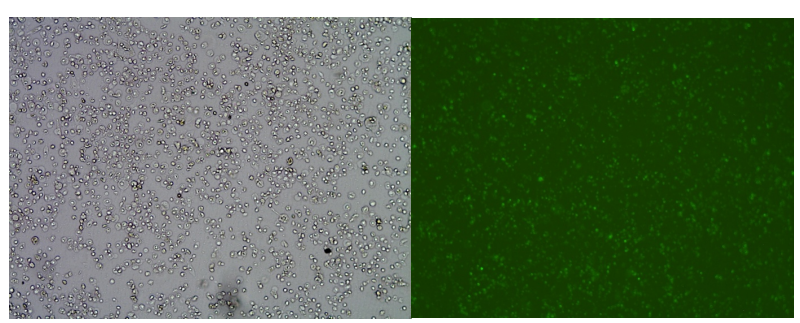

Figure 13. The Transfect Efficiency of Cell Under Fluorescence Microscope

respectively. The difference was statistically significant (Figure 11 and Figure12).

Lipofectamine $e^{T M} 2000$ can be used for transfect cervical cancer cells

As the optimal transfection conditions of the above experiments :The seeding density of Hela cell line and Siha are $1.5 \times 10^{4}$ (per well of 24 well plates), miRNA amount is $1 \mathrm{ul}$, the ratio of miRNA and liposome is 1:0.5 of Hela cell line; 1:0.7 of Siha cell line respectively, after 24 hours we can observed transfection results by fluorescence microscopy.Inverted fluorescence microscope at a magnification of 4 times of the eyepiece $\times 10$ times of lens. Visible that the successfully transfected cells emit green fluorescence (Figure 13). 


\section{Discussion}

Cationic liposomes as transfection medium, has the characteristics of the following aspects: 1) belong to non-reactive sterically stabilized liposomes, has a low rate of recognition and ingestion by the immune system. 2) The formation of DNA / liposome complexes can not limited by the size of DNA. 3)Having a higher transport capacity for DNA which with a high charge. 4) it also can transfer RNA, ribosomal and other large charge molecules and macromolecules into the cells, The transfection efficiency is higher (Ma et al., 2015) (He et al., 2010). However, it also has some limitations. Such as the nonuniformity, degradability and dynamic change of transfection complexes will gain a lower efficiency than viral transfection (Kong et al., 2015). Therefore, how to improve the liposome transfection efficiency has become the research hotspot.

Maintaining the status of transfected cells to vigorous growth and health that is the fundamental condition to improve the transfection efficiency (Alimohammad Asgharian et al., 2014). Therefore, controlling the concentration of inoculated cell has a certain impact on transfection efficiency. Under the conditions of this experiment, cells were plated at $1.5 \times 10^{4}$ per well of 24 well plates is the suitable inoculation density.

In this experiment where Lipofectamine ${ }^{\mathrm{TM}} 2000$ amount unchanged, The transfection efficiency of showed a trend of increase with the increase of miRNA dosage. However, when the quantity of miRNA more than 1ul in per well of 24-well plate ,the transfection efficiency is in the doldrums. It may be because when all miRNA combined with liposome, the continuous miRNA won't form excess miRNA-liposome complexes. Instead, high doses of miRNA would be a waste.

Theoretically speaking, Liposome with positively charged ions and nucleic acid which with negatively charged, Both the proportion of 1:1 or above, nucleic acid transporter works best (Fujii Y et al., 2010). In this experiments, we found that the ratio of miRNA and liposome is 1: 0.5 of Hela cell and the ratio is 1: 0.7 of Siha. Excessive liposomes are toxic to cells, which led to a decline in transfection efficiency (Hasenkamp $\mathrm{S}$ et al., 2012). Furthermore, we found that different kinds of cell type maybe the other one major factors which influence general transfection ability (Chandramouli A et al.,2012): Hela cell is known to proliferate rapidly, however in our study, which are more sensitive to the toxic effects of liposomes, The reason may be the typical desmosomes in connection area and the abundant tension wire in cytoplasm of Siha cells which makes it has a relatively weak toxic for liposomes (Wolfram J et al.,2014). But the exact reason of its difference is not known .

Transfection time is one of the conditions that affect transfection efficiency (Park et al., 2015). In general, transfection time and transfection efficiency should be a positive correlation. In one aspect, liposomes are toxic to cells. on the other hand, the culture medium is in the absence of serum when the cells transfected. The experimental results show that when the reaction time of miRNA/ liposomes compounds is less than 6 hours, the transfection efficiency is low because it haven't fully embodying the exogenous gene of the cell. The transfection time was extended to 6-12 hours, can improve the transfection efficiency. After 24 hours, under the microscope we can see a lot of dead cells floating in the medium because of the enhanced toxicity of liposomes, that is to say the transfection efficiency were decreased.

The serum in the culture medium can also affect the transfection efficiency (Jin et al., 2014). In this experiment, before the cells transfected remove serum to Siha cells, the transfection efficiency was significantly changed, but there was no statistically significant difference between two groups of results in Hela. In serum, the proteins with the negatively charge can nutrient the liposome complexes who with the positively charged, Bring down the transfection efficiency (Un K, 2012). Thus when we prepared liposome / miRNA complexes ,the diluent can not add with serum, 6 hours after transfection, replace this medium, We can be added to a medium which containing $10 \%$ FBS. It is little impact on transfection efficiency at this time. Join serum can improve cell viability, reduce cell damage caused by hunger, maintain a relatively healthy cell morphology (Soto-Sanchez et al., 2015).

The experiment we used Hela cell line and Siha cell line, from seeding density, the amount of miRNA and its proportion of liposomes, transfection time, the presence or absence of serum and cell toxicity experiments etc to explore how to improve the efficiency of transfection of cervical cancer cells line. It can be suggested that Liposomes is an appropriate reagent which use miRNA to transfect into Hela cell and Siha cell, and the rate of expression of a candidate gene can be evaluated easily after transfection by using this reagent. Through this experiment, it can help to provide assistance for further research which in miRNA regulatory mechanism in cervical cancer in the future.

\section{Acknowledgements}

This research is supported by Xinjiang Uygur AutonomousRegion Natual Science Foundation (No. 2015211C008).

\section{References}

AAsgharian A, Banan M, Najmabadi H (2014). Optimizing a lipocomplex-based gene transfer method into hela cell line. Cell J, 15, 372-7.

Alimohammad Asgharian, Mehdi Banan, Hossein Najmabadi (2014). Optimizing a lipocomplex-based gene transfer method into hela cell line. Cell J, 15, 372-7.

Chandramouli A, Onyeagucha BC, Mercado-Pimentel ME,et al (2012). MicroRNA-101 (miR-101) post-transcriptionally regulates the expression of EP4 receptor in colon cancers. Cancer Biol Ther, 13, 175-83.

Fujii Y, Kachi S, Ito A et al (2010). Transfer of gene to human retinal pigment epithelial cells using magnetite cationic liposomes. Br J Ophthalmol, 94,1074-7.

Gaedcke J, Grade M, Camps J,et al (2012). The rectal cancer microRNAome-microRNA expression in rectal cancer and matched normal mucosa. Clin Cancer Res, 18, 4919-30.

Geusens B, Lambert J, De Smedt SC, et al (2009). Ultradeformable 
cationic liposomes for delivery of small interfering RNA (siRNA) into human primary melanocytes. J Control Release, 133, 214-20.

He ZY, Zheng X, Wu XH,et al (2010 ). Development of glycyrrhetinic acid-modified stealth cationic liposomes for gene delivery. Int J Pharm, 397, 147-54.

Hasenkamp S, Russell KT, Horrocks P (2012). Comparison of the absolute and relative efficiencies of electroporationbased transfection protocols for Plasmodium falciparum. Malar J, 11, 210.

Jin M, Yang Z, Ye W,et al (2014). MicroRNA-150 predicts a favorable prognosis in patients with epithelial ovarian cancer, and inhibits cell invasion and metastasis by suppressing transcriptional repressor ZEB1. PLoS One, 9, 103965.

Kong Q, Wu G, Han L,et al (2015). A transfection method of PS-asODNs targeting ANGPTL4 in multicellular structures of hepatocarcinoma cell line. Cancer Gene Ther, 22, 285-90.

Liang X, Potter J, Kumar S,et al ( 2015). Rapid and highly efficient mammalian cell engineering via Cas9 proteintransfection. $J$ Biotechnol, 208, 44-53.

Lee WM, Chen Y, Wang W, et al (2015). Growth of human rhinovirus in H1-HeLa cell suspension culture and purification of virions. Methods Mol Biol, 1221, 49-61.

Ma X, Lin Y, Yang K, et al (2015). Effect of lentivirus-mediated survivin transfection on the morphology and apoptosis of nucleus pulposus cells derived from degenerative human disc in vitro. Int J Mol Med, 36, 186-94.

Pradines B, Lievin-Le Moal V, Vauthier C, et al (2015). Cell line-dependent cytotoxicity of poly (isobutylcyanoacrylate) nanoparticles coated with chitosan and thiolated chitosan: Insights from cultured human epithelial HeLa, Caco2/TC7 and HT-29/MTX cells. Int J Pharm, 491, 17-20.

Park E, Cho HB, Takimoto K (2015). Effective gene delivery into adipose-derived stem cells: transfection of cells in suspension with the use of a nuclear localization signal peptide-conjugated polyethylenimine. Cytotherapy, 17, 536-42.

Soto-Sanchez C, Martinez-Navarrete G, Humphreys L, et al (2015). Enduring high-efficiency in vivo transfection of neurons with non-viral magnetoparticles in the rat visual cortex for optogenetic applications. Nanomedicine, 11, 835-43.

Takahashi RU, Miyazaki H, Takeshita F, et al (2015). Loss of microRNA-27b contributes to breast cancer stem cell generation by activating ENPP1. Nat Commun, 6, 7318.

Un K (2012). Development of cell-selective gene transfection method using sugar-modified and ultrasound-responsive liposomes. Yakugaku Zasshi, 132, 1273-9.

Wolfram J, Suri K, Huang Y, et al (2014). Evaluation of anticancer activity of celastrol liposomes in prostate cancer cells. J Microencapsul, 31, 501-7.

Xiong F, Mi Z, Gu N (2011). Cationic liposomes as gene delivery system: transfection efficiency and new application. Pharmazie, 66, 158-64. 